\title{
Empleabilidad de la generación Millennials
}

\author{
Employability of the Millennials Generation
}

\author{
Pedro Melchor-Chávez ${ }^{a}$, Ivette Flores-Jiménez ${ }^{b}$, Raúl Rodríguez-Moreno ${ }^{b}$, Miguel A. Vázquez- \\ Alamilla $^{b}$, Ruth Flores-Jiménez, ${ }^{b}$
}

\begin{abstract}
:
Throughout this work are described the main characteristics and most relevant event of the generation named "Millennials" in the laboral scene, distinguish some aspects related to their formation, growth, education and work to understand how they learn of the current globalized world and digitized in a highly competitive way. This generation, which starts from the 80's, they are people which interacted with the great technological changes of the world that occurred in less than 20 years and they got to adapt without problem, although they also remember the world before all the boom of technology and internet. They are entrepreneurs by nature since they consider that hard work and determination are the key to success in these times.
\end{abstract}

Keywords:

Millennials, globalization, job

\section{Resumen:}

A través de este trabajo se describen las principales características y acontecimiento más relevantes de la generación denominada "Millennials" en el ámbito laboral, destacando algunos aspectos relacionados con su formación, crecimiento, educación y trabajo para poder entender cómo es que aprenden del actual mundo globalizado y digitalizado de una manera altamente competitiva. Dicha generación, la cual comienza a partir de la década de los 80, son personas las cuales interactuaron con los grandes cambios tecnológicos del mundo que ocurrieron en menos de 20 años y ellos lograron adaptarse sin problema, aunque también recuerdan el mundo antes de todo el boom de la tecnología e internet. Son emprendedores por naturaleza ya que consideran que el trabajo duro y la determinación son la clave del éxito en estos tiempos.

Palabras Clave:

Millennials, globalización, trabajo

\section{Introducción}

La presente investigación hace referencia al tema de la "generación Y" o también conocida como "Millennials" es decir, el grupo de personas nacidos entre 1980 y 2000 , que es importante señalar que los diferentes investigadores no han especificado con exactitud las fechas donde comienza y termina el nacimiento de esta generación, lo que sí están seguros es que han crecido con la existencia de Internet. Actualmente se sabe que las generaciones anteriores a estas son: La generación tradicional (personas nacidos antes de 1945), la generación Baby boomers (personas nacidos entre 1945 a 1964), la generación "X" (personas nacidos entre 1965 a 1980) y la generación" $Y$ " (personas nacidos entre 1981 a 2000) por último la generación "Z" que está empezando a intervenir en la economía del mundo. (Fall, 2009) Esta generación se caracteriza por que los jóvenes que la integran son: optimistas, seguros de sí mismos, trabajadores en equipo y étnicamente diversos esto refriere a lo positivo y a lo negativo, personas poco acostumbradas al esfuerzo, poca seriedad en el trabajo y también son considerados como narcisistas. Esta generación está altamente alfabetizada en temas tecnológicos, pues crecieron en una época donde las comunicaciones a través de internet estaban en su pleno

Autor de Correspondencia, Universidad Autónoma del Estado de Hidalgo, Escuela Superior de Tlahuelilpan, Email: pedro_melchor6320@uaeh.edu.mx

b Profesor Investigador, Universidad Autónoma del Estado de Hidalgo, Escuela Superior de Tlahuelilpan. Email: Ivette Flores Jiménez: ivette_flores7@hotmail.com; Raúl Rodríguez Moreno: raromo@hotmail.com; Miguel Ángel Vázquez Alamilla: m_vazquez_alamilla@hotmail.com; Ruth Flores Jiménez: ruthfj_22@hotmail.com 
apogeo. El uso de las redes sociales, mensajería móvil, chat y juegos en red, es común verlo entre estos jóvenes. Para analizar el tema es indispensable saber que el desarrollo de las nuevas tecnologías facilita el acceso al conocimiento y al aprendizaje, esto no pasaba en generaciones anteriores. En el ámbito educativo, es muy común observar a los profesores de mayor experiencia, una cierta preocupación ante la posibilidad de que los estudiantes lleguen con más conocimiento que el propio docente, más, sin embargo, los docentes que se han adaptado a estas nuevas tecnologías observan, con asombro, cómo este grupo de personas puede llegar a conocer muchas herramientas, pero es preocupante su uso inadecuado, crea un gran problema en el entorno personal y en el profesional.

Los millennials usan internet para comunicarse, entretenerse, buscar información, crear y participar en determinada actividad. Las experiencias de aprendizaje informal pueden surgir desde actividades como grabar videos, realizar trabajos artísticos, escribir y compartir historias, hacer composiciones, entre otros.

Esta generación es única y diferente a generaciones pasadas por que son desafiantes ante los retos del futuro, son una generación digital, muy bien conectada y con altos valores sociales y éticos. Las empresas, si quieren conquistarles y mantener el talento entre sus filas, deben atender a estos datos donde valoran la flexibilidad, el trabajo desde casa y el reconocimiento no por años, sino por sus funciones. Las organizaciones que no sepan dar respuesta a estas peticiones perderán parte del personal más joven.

La investigación de este fenómeno social se realiza con el propósito de determinar las características, cambios, formas de pensar y el FODA que posee la generación " $Y$ " o también llamados generación del milenio.

\section{A. Planteamiento del problema}

La dificultad que presentan los millennials para incursionar en el sector laboral.

\section{B. Objetivos específicos}

Caracterizar el comportamiento actual de la generación milenaria o generación " $Y$ " en el trabajo.

Determinar el tipo de expectativas en el trabajo tienen los jóvenes que forman parte de esta generación.

Identificar las actitudes y aptitudes que presentan los millennials en el campo laboral.

C. Hipótesis
La generación "Millennials" sufre inestabilidad laboral en gran parte debido al modo de emplearlos que predomina, por lo que busca un trabajo independiente, no asegura un empleo a largo plazo dentro de una empresa, provocando un desplazamiento constante y de poco interés. Debido a que dicha generación se preocupa mayormente por su desarrollo personal sobre el laboral, pero también tienden a emprender de forma constante al considerar esta última la base del éxito actual y futuro.

\section{Marco Referencial}

\section{A. Millennials}

Los Ilamados Millennials son una generación digital, muy bien conectada, con grandes valores éticos y también morales. Son la parte de la población que se resiste a las empresas, también compiten mucho en el sector, al mismo tiempo los Millennials son creadores de contenido e influyentes en el sector en el que se encuentran.

Los Millennials han hecho posible atender varios dispositivos a la vez. Una parte muy grande del sector de los Millennials tienen una preparación alta porque son de grado superior. Pero, en los últimos tiempos se menciona que los Millennials no solo quieren obtener títulos universitarios y un trabajo que sea estable, sino que, ellos quieren ser emprendedores y por eso se convierten en personas muy capaces con grandes ideas novedosas, que inclusive son premiadas.

Los mismos Millennials son desafiantes ante los retos que se presentan a futuro, lo que ellos buscan en las organizaciones es que se les reconozca por su esfuerzo y no por el tiempo que trabajan en la empresa, de igual manera que valoren la flexibilidad con la que cuentan.

El problema que tiene esta generación se encuentra más allá de no estar satisfechos con sus objetivos laborales, en muchas ocasiones tienen problemas para encontrar un empleo fijo, al igual que independizarse, derivado de que no tienen una buena situación económica, muchos de ellos aún viven con sus padres. También son incrédulos ante cierto tipo de puestos en las organizaciones.

Una gran virtud que caracteriza a los Millennials es que tienen un sentido crítico y exigente, cuentan con colaboración, transparencia, tolerancia y compromiso, y estos son algunos de los valores que ellos quieren ver reflejados en la sociedad, cuentan con una gran conciencia acerca de la situación que se vive a nivel mundial, de igual manera comprenden que el futuro económico y político acabará en sus manos y por tanto tienen que ser responsables de sus acciones.

Por esta situación muchos de los movimientos que luchan por la incorporación social son encabezados por los Millennials, por lo tanto, muchas iniciativas tienen una 
gran participación de jóvenes que están involucrados directa o indirectamente.

Algunos de los rasgos clave que los caracterizan es que son volátiles, versátiles y volubles, de esta manera podemos entender su comportamiento, esto da como consecuencia el aumento de individualidad.

En un entorno tan globalizado los Millennials buscan diferenciarse y destacarse de los demás, teniendo al alcance las mismas herramientas como son eventos y cultura, redes sociales, cadenas de ropa, es por ello que esta generación es creadora y consumidora de contenido, buscan destacar y ser reconocidos, ya que este es uno de sus valores más importantes para esta generación.

Es fácil vincular todas las características que distinguen a esta generación y que la hacen única a través de la individualidad, están utilizando los avances tecnológicos que se encuentran a su alcance para que de esta manera puedan especializarse y así encontrar un trabajo que satisfaga sus necesidades y que también divulgan sus ideas políticas y sociales que no solo buscan la tolerancia, sino también la igualdad.

Los Millennials retrasan etapas de la vida adulta, como son el matrimonio y la vida laboral. Son la generación más diversa, más conectada y con un alto nivel educativo. .

La vida de esta generación es móvil y la principal ventana al mundo es la red, son extremadamente sociales, los Millennials se están preparando para trabajar durante toda su vida. Se han vuelto más críticos y exigentes ya que las circunstancias los han obligado a ser así, para que de esta manera se obtengan mejores resultados en la vida personal como en la vida empresarial.

En esta década por lo general no les gusta ser llamados Millennials, ya que con este significado suelen ser catalogados como perezosos, o que de la misma forma no les gusta trabajar.

Existe una gran diferencia de un individuo hacia otro, por lo cual no pueden ser identificados, e incluso sus características son mayores a las generaciones anteriores. Esta generación ha ido creciendo en una sociedad completamente diferente a las anteriores. Esta generación ha ido desarrollándose tecnológicamente desde que eran niños, son una generación segura.

Es la generación simplemente más diversa, y suelen ser tolerables a la diferencia. Siendo educados con la frase de "alcanza tus sueños" y diciéndoles que son especiales, ellos suelen ser muy seguros de sí mismos, a causa de esto se provoca mucha seguridad en sí mismo. Son más optimistas sobre el futuro y sus países que otras generaciones.

Arrojando los resultados obtenidos, es que estos entran a la edad adulta con expectativas un poco irrealistas. La mayoría entró a la universidad solo para encontrarse con trabajos que no querían realizar en un principio, cuando las generaciones pasadas esperaban un poco más. Sus expectativas son muy alentadoras e involucradas.

Son conocidos en el lado negativo por ser perezosos, enamorados de sí mismos y que no pueden mantener un trabajo ya que van de un trabajo a otro, siendo inestables en el campo laboral. Estos desean un horario de trabajo más relajado a modo de que puedan tener más tiempo para ellos, que sea flexible. Otra de las características es que suelen ser menos comprometidos y más irresponsables con lo que respecta al civismo y la política, y más focalizados en su personalidad. En su mayoría son personas que no se preocupan ni les preocupara en ayudar a la comunidad como lo es la generación $X$ cuando tenían la misma edad, e incluso están más focalizados en dinero, fama en vez de interesarse en valores intrínsecos o aceptación propia.

Por otro lado, suelen ser de más mente abierta, y aceptan más los derechos homosexuales ya que pertenecen al grupo que apoya la igualdad de derechos para la minoría. Las características positivas de esta generación es que suelen ser muy seguros de sí mismos, expresivos, liberales y receptivos en cuanto a nuevas ideas y formas de vivir y a la misma vez e innovar.

Lo que es cierto la generación $X$ fue desarrollada mayoritariamente en la década de la comunicación digital: internet, telefonía móvil y redes sociales. Esto arroja que sí es correcto afirmar que los Millennials tienen un manejo diferente e inteligente en las tecnologías de la comunicación.

Los integrantes pertenecientes a esta generación poseen una personalidad única, daño a demostrar así su preponderancia en la cultura, esto los conlleva un pensamiento más estratégico, además de que son más sociables y pueden contar con una actitud de más positiva y seguros de sí mismo, el tener tiempo y paciencia para realizar varias actividades a la vez y la tecnología les permite poder lograrlo. Para ellos es de suma importancia su autonomía y a su libertad.

En su ideología piensan que todos deben tener su propia opinión, que sea escuchada y respetada independientemente de su edad, su experiencia y sexo. Mientras los gerentes encanecen y trabajan jornadas cada vez más largas, los Millennials (la generación nacida entre 1980 y el 2000) se incorporan a la fuerza laboral a un ritmo acelerado en los últimos años. En la convivencia, las dificultades de comunicación no se han hecho esperar, la nueva generación trae consigo diferentes formas de trabajo y de relación con sus compañeros de trabajo y jefes inmediatos, son empleados menos leales que generaciones anteriores.

Esto ocurre porque es la naturaleza de esta generación que los lleva a cambiar de puestos de trabajo distintas 
veces en su vida, con el fin de encontrar en un entorno de aprendizaje continuo y en el que nuevos retos sean constantes, algo que es negativo y no podría encajar en las empresas.

La solución es encontrar maneras productivas de trabajar y establecer puentes que superen el abismo en la comunicación, esta generación valora aspectos como: la flexibilidad laboral, la formación continua, el trabajo colaborativo y por objetivos, la imagen de marca de la compañía para la que trabajan y su reputación, esta generación está más comprometida con la sociedad que con sus predecesores por lo que aquellas empresas que no tengan políticas socialmente responsables tendrán que enfrentarse a un incremento en la rotación , las organizaciones que lo hagan tendrán grandes ventajas estratégicas sobre los demás.

Cuando los gerentes o jefes de áreas encargan de motivar y dirigir a esta generación ,como evaluar candidatos y asegurar que sean buenos elementos para el equipo, como expresar expectativas y lograr resultados. (Caraher)

La generación Millennials busca innovar pues ya que uno de sus principales cambios que ha enfrentado esta generación ha sido en el campo laboral.

Consigo esta generación a desarrollado nuevas economías. Está nueva economía es conocida como la economía del conocimiento y necesita de nuevos profesionales y personas de alto rendimiento o altamente competitivas. Es una economía basada en la tecnología, la gestión de datos y la innovación de nuevos productos. Una economía que está cambiando fuertemente y radicalmente todas las industrias que de conocen en mayor o menor medida.

También es conocida como economía de la atención dado que nuestro principal recurso es el tiempo, que tenemos que gestionar de la manera más eficiente que sea posible.

Sin duda alguna nos encontramos en un contexto complejo para las empresas, el cual tienen que manejar con mucho cuidado. Junto a las prioridades de los Millennials conviven las necesidades totalmente distintas de las generaciones anteriores, los Baby boomers y la generación $x$ menos comprometidas socialmente, son más fieles a una marca o empresa y al talento y experiencia que aportar al sector empresarial. Esto ha implicado un gran cambio de fuerzas que dominan en la economía.

Los Millennials cambiarán los paradigmas del comercio junto con hábitos de consumo diferentes, mayores exigencias y diferentes gustos, así mismo como preferencias y tendencias distintas a las que predominan hoy en día en cuanto a la oferta de productos y servicios. Las experiencias durante el tiempo de estudios y en primeros puestos de trabajo, pasan de ser una experiencia propia enriquecedora a ser un requisito indispensable para la empleabilidad en años actuales. Un dato muy importante es el saber que durante tu preparación y desarrollo laboral, tienes que considerar que quienes no tengan un buen manejo de alguna lengua internacional no podrán aspirar a ningún puesto de gran relevancia o ni a ingresos altos, sin importar el desempeño que poseías durante tu tiempo de estudios o experiencia en algunas otras empresas, el saber hablar una lengua internacional abrirá muchas espacios. (Haro, 2017)

Se estipula que son la generación mejor educada y que se les conoce por que esta generación evita riesgos económicos, pues no se comprometen a manejar una tarjeta de crédito, comprar carro o incluso una casa, la mayoría aún vive con sus padres, por la buena relación que llevan con sus padres, pero en esta generación afecta la crisis que hubo pues ya que no con facilidad encuentran trabajo y gasta más de lo normal, están menos asociados con las instituciones religiosas, políticos, incluso hasta para el matrimonio, apuestan por el empleo creativo, pues buscan placer, solo trabajan en algo que les guste y disfruten". (Mabel, 2014)

Esta generación se considera que va ser la que más va vivir y va a trabajar, es la que va enfrentar un mercado laboral en transformación, porque los empleos van hacer de larga duración y con mejor estabilidad, ya que estas personas Ilamadas Millennials no recibirán "pensión del gobierno", por lo que ahora van a tener muy en cuenta en ahorrar por cuenta propia para su vejez, y esto está claro que no se tenía pensado, pues siempre había existido la pensión.

Esta generación prioriza a la hora de elegir donde trabajar ya que les gusta estar en capacitaciones constantes, seguir estudiando par ser mejor cada día y así mismo necesita de motivación y compromiso en el ambiente laboral, para así sentirse exitosos y estar preparados para su empleo y para que tengan un mejor salario.

"Los millennials se preparan para trabajar toda su vida, el $36 \%$ espera hacerlo hasta después de los 65 años, el $19 \%$ se proyecta trabajando después de cumplir los 70 , y el $8 \%$ dice que lo hará hasta el día de su muerte", según (Forbes, 2016).

\section{B. Características de los Millennials}

Esta generación está adaptada a la tecnología. Son emprendedores y creativos intentan vivir de los que aman hacer, como viajar, conocer el mundo y subir las fotos a las redes sociales.

Son caprichosos, no soportan los compromisos, refieren estar solos, prefiere una mascota o una colección de likes en Facebook. 
Destacados, optimistas. Coaching, motivacional, colaborativo. Reconocen sus metas personales. Buena comunicación (Macarena, 2015)

\section{Generación Baby Boomers}

Nacidos en los años 1945 y 1964, tras la segunda guerra mundial, la mentalidad de esta generación de tener una buena estabilidad, ser un buen empleado con estructura jerárquica, tener una oficina, horario fijo y trabajar muy duro para tener una casa para darle seguridad a su familia (Mabel, 2014).

A esta generación les gusta que el trabajo sea estable a largo plazo y es adicto a él, no le dedican mucho tiempo al ocio y a la actividad recreativa. Orientados al servicio, dedicados, definen la misión del grupo. Esperan como recompensas: promociones y reconocimiento formal. Tienen buena comunicación (Macarena, 2015).

\section{Generación X}

Nacidos entre 1961 y 1981, es la generación que vio caer el muro de Berlín, esta generación trataba de sobresalir en el trabajo y tener en corto tiempo el mayor crecimiento laboral, llevan sus labores más allá de la oficina, era muy autosuficientes, no se preocupan por arreglar y lo que la gente piense de ellos (Mabel, 2014).

Los hombres y las mujeres de la generación $\mathrm{X}$ trabajan mucho pero logran un equilibrio, no les incomoda o les hace infeliz trabajar mucho, son felices con sus propias vidas. Son los que vieron la creación del internet y los avances tecnológicos. Están adaptados a los grandes cambios sociales. Son más propensos a estar empleados pues aceptan las reglas tal cual son, están equilibrados entre el trabajo, los hijos y el tiempo para el ocio. Son flexibles y adaptables, buscan un balance entre trabajovida personal. Son independientes y emprendedores (Macarena, 2015)

E. Generación Z.

Nacidos entre los años de 1995 y hasta el presente. Son verdaderamente nativos digitales, porqué desde pequeños están conectados con la tecnología, usan internet. Esta generación es autodidacta, aprenden por medio de tutoriales. Son creativos ya que incorporan rápido nuevos conocimientos y son muy sociables, se relacionan bien. Se destacan porque siempre están sobre-informados, tienen una alta propensión al consumo de información y entretenimiento.

Visitan redes que sus padres no, como el Snapchat, Instagram, porque en estas redes sociales comparten contenido de su vida privada, e aspiran a ser YouTubers, su vida social pasa en un alto porcentaje por las redes sociales. Pasan mucho de su tiempo frente a las pantallas. Su éxito se mide en compartidos y likes.
Todavía no acceden a la vida laboral, pero se estipula que les preocupa encontrar una vocación o algún trabajo acorde a sus gustos, conocerse a sí mismos y aceptar las diferencias en un mundo más globalizado. Aprecian los valores empresariales tradicionales, prefieren trabajar en empresas grandes que puedan responder a sus necesidades. En esta generación están dispuestos a trasladarse a otro estado por una oferta de trabajo (Rebolledo, 2017).

F. FODA generación Millennials. Fortalezas:

- Son nativos digitales.

- Habilidad para manejar las Tics.

- Se adaptan a los cambios.

- Son sociables.

- Seguridad en sí mismos.

- Libertad de decisión.

Debilidades:

- Falta de compromiso en actividades con respecto al trabajo.

- Son dependientes de sus padres

- Si no sienten que son tomados en cuenta, o que no les responden como ellos quieres, abandonan su trabajo.

- Son irracionales.

Oportunidades:

- Adelantos tecnológicos en temas de comunicación.

- Existencia de dispositivos móviles inteligentes.

- Existencia de herramientas digitales para realizar actividades laborales.

\section{Amenazas:}

- Altos niveles de desempleo.

- Falta de acceso a internet en algunas regiones del país.

- La mayoría de las empresas piden experiencia laboral.

\section{Metodología}

El trabajo cumple con las características de un enfoque cuantitativo comenzando por el planteamiento de un problema de estudio delimitado, que deriva a una hipótesis que se pretende comprobar a partir de la recolección de datos a través de encuestas aplicadas. Investigación práctica cuantitativa ya que se centra en aspectos medibles numéricamente de los fenómenos y se sirve de pruebas estadísticas para el análisis de los datos que expliquen el fenómeno a través del método deductivo (Hernández Sampieri, 2014). 
Investigación transversal dado que se refiere a un momento durante el ciclo escolar enero - julio del año 2018 y de la situación específica en la Escuela Superior de Tlahuelipan. Descriptiva dado que se refiere a la descripción los temas de empleabilidad entre los alumnos pertenecientes a la generación de Millennials. Se desarrolla en campo, dado que se realiza en las instalaciones de la institución en situaciones naturales, que permite la generalización en situaciones similares pero, sin un control riguroso del estudio en cuestión.

\section{Resultados}

Tras la obtención de datos a través de la encuesta a la muestra de 64 personas, todas estas alumnos de la licenciatura en administración de la Escuela Superior de Tlahuelilpan, los cuales se encuentran entre los 34 y 18 años, se lleva a cabo el análisis de los mismos, donde se afirmarán o impugnarán los objetivos marcados para con esta investigación.

1. ¿Cuántos años tienes?

Esta pregunta inicial fue para saber el rango de edad en las que se encuentran los alumnos de esta escuela superior, y con un $8 \%$ se encuentra en la edad de 28 a 34 años, con un 19\% está en un rango de 23 a 27 años y con un $73 \%$ se encuentra en edad de 18 a 22 años.

2. ¿Qué tanta importancia le das al sueldo, al aceptar una oferta laboral?

Para los millennials el salario es un factor motivador, y algunos son los que le dan mayor importancia, pues muchos de estos millennials no les pagan como debería por la falta de experiencia laboral, y en esta encuesta el $8.12 \%$ le dan poca importancia al salario, no les interesa ganar un sueldo atractivo y el $57.88 \%$ les importa mucho el sueldo.

3. ¿Qué tanta importancia le das al horario, al aceptar una oferta laboral?

Para los millennials el horario de trabajo es importante ya que enfrentan el reto de adaptarse al cambio, sin embargo al realizar la encuesta se destaca que el $2.3 \%$ no le dan importancia al horario de trabajo, el $30.47 \%$ les es muy importante y el $32.50 \%$ les es importante el horario de trabajo al aceptar una oferta laboral.

4. ¿Qué tanta importancia le das al renombre de la empresa, al aceptar una oferta laboral?

La generación Millennial le da mucha importancia al renombre de la empresa, porque se necesita saber si la organización tiene una buena imagen en el mercado. Según las respuestas de los millennials el $4.6 \%$ no les interesa saber el renombre de la empresa, con un $27.42 \%$ les interesa saber poco y el $33.52 \%$ le dan mucha importancia al renombre de la empresa.

5. ¿Qué actividades creativas consideras que hace más interesante a una empresa?

Muchas veces las actividades creativas para las empresas son importantes, ya que con estas actividades se les puede bonificar o premiar al personal de la empresa, que se les tome en cuenta o tengan ratos de actividades para despejar un poco la mente, por lo que para esta generación consideran que las actividades creativas más interesantes son las dinámicas para empleados

6. ¿Cuál son los objetivos a corto plazo?

La generación busca continuar estudiando al darse cuenta de la cantidad de competencia que existe hoy en día, necesitan prepararse y obtener mayos conocimiento para poder llegar a ser competentes.

7. ¿Cuáles con tus objetivos a largo plazo?

De igual manera en esta pregunta la generación sigue con la idea de continuar fortaleciéndose con nuevos conocimientos, están conscientes que conforme trascienden los días el mundo cambia radicalmente con nuevas tecnologías e innovaciones a las que se es necesario adaptarse. Los objetivos que tienen los Millennials a largo plazo son más concretos al darse cuenta que tienen que ser cada vez más objetivos.

8. ¿Cuántos años te gustaría permanecer trabajando en una misma empresa?

Los Millennials que fueron encuestados, consideran que es mejor para ellos permanecer laborando un buen tiempo en alguna empresa, pues dentro de al pasar un tiempo pueden ascender de puesto e innovar dentro de ella.

9. ¿Cuáles son los atributos que buscas en la compañía para trabajar?

La mayoría de los encuestados buscan liderazgo de excelencia dentro de la empresa, pues consideran que es muy importante desarrollarlo dentro del ámbito laboral y personal.

10. ¿Te gusta que te llamen Millennials?

Las personas encuestadas dentro de este proyecto, son de la universidad y generaciones que entran dentro del margen de edad de los millennials. De lo que destaca que la mayoría no tiene idea que pertenece a esa generación o simplemente que es millennials.

\section{Discusión}

El mercado laboral para esta generación se reviste de un mayor grado de exigencia, en capacitación, formación, habilidades y conocimiento, también hay otra comparativa en relación a los datos de desempleo y las limitaciones para acceder a un trabajo decente, pues es 
una realidad que coexiste, pero que no tiene la misma atención y posicionamiento en este debate. Los millennials, comparan y visualizan posición de trabajo, que les pueda dar una calidad de vida diferente a las que las otras generaciones están acostumbradas, pues los millennials buscan gozar de bienes, aunque no sean propios, buscan experiencias, viajes, comprar felicidad, y buscan un empleo que les de estas posibilidades.

Se debe entender que los denominados millennials como tal, presentan diferencias con respecto a generaciones anteriores, en cuanto a valores, estilos de trabajar, creencias, cultura y especialmente en su modo de aprendizaje ya que este nada se compara con los métodos que se ocupaban en las generaciones anteriores, puesto que con la llegada del internet hace ya varios años, este ha facilitado la vida de las personas, principalmente en el estudio y en el trabajo.

\section{Conclusiones}

Se piensa que los millennials son poco rentables para las grandes empresas por los prejuicios que se les han asignado, ya que se habla de una generación floja, poco comprometida, sin embargo no todo es malo, también hay cosas buenas. Aunque para la sociedad en general son pocos los millennials sobresalientes.

Los millennials pueden ser personas con buenas ideas, compromisos y buenos líderes, si se lo proponen, saben usar la tecnología, usan internet para comunicarse, entretenerse, buscar información, crear y participar en determinadas actividades.

Se caracterizan porque las personas que integran esta generación son optimistas, seguros de sí mismos, trabajadores en equipo, acatadores de las reglas que se deben de seguir, poco acostumbradas al esfuerzo, ya que cambian mucho de trabajo por no cumplir con la expectativa que esperan, poca seriedad a la hora de trabajar, se lo quieren llevar todo siempre al relajo, quieren seguir la costumbre del típico mexicano "hay mañana", todo a la mera hora y también son considerados como narcisistas, piensan que todas las cosas son fáciles ya que en su tiempo de niñez tenían todo a la mano.

La solución para esta generación es encontrar maneras productivas de trabajar y tener más comunicación, ya que valorarán aspectos muy importantes, como la flexibilidad laboral, la formación continua, el trabajo colaborativo y por objetivos, la imagen de marca de la compañía para la que trabajan y su reputación, así solo podrán comprometerse con ellos mismos y con la organización, solo de esta manera podrá salir a flote la organización. Esta generación está más comprometida con la sociedad que sus predecesores, por lo que aquellas empresas que no tengan políticas socialmente responsables tendrán que enfrentar un incremento en la rotación, la organización que lo haga tendrá grandes ventajas estratégicas sobre las demás.

Esta generación por lo general no les gusta ser llamados Millennials, algunos no saben que son Millennials ni su significado, ya que saben que suelen ser catalogados como perezosos o que no les gusta trabajar.

Existe una gran diferencia de una persona hacia otro y es por eso que se llega a la conclusión de que no todos los millennials son así, con características de malos o buenos. Cada persona piensa diferente, por lo que no pueden ser identificados de forma absoluta, incluso sus características son mejores a las de las generaciones anteriores, debido a que esta ha creciendo en una sociedad completamente diferente, con características diferentes que los hacen ser únicos. Esta generación ha ido desarrollándose tecnológicamente desde que eran niños apoyados por sus padres, que los hace ver de otra forma el mundo, más fácil.

Ser un Millennial en este tiempo es ser una persona con pensamientos muy diferentes a otras, hasta con los de su misma generación, es por ello que cada quien sabe las responsabilidades y su modo de pensar, según como quieran ver el futuro, como quieran que sea su vida, como quieran que los vean para así ser tratados.

Es importante hacer notar que se percibe un desfasamiento en la temporalidad de la generación Millennials, con respecto a la de las grandes ciudades de México y de otros países, que podría ser de entre cinco y diez años. Lo que puede ser debido a la forma en la que se va desplazando la ola tecnológica en las distintas localidades alrededor del mundo, sobre todo en las comunidades más rezagadas social y económicamente.

\section{Glosario}

SALSEO: Cualquier polémica, discusión o controversia llevada a cabo en las redes sociales será considera un salseo. Es uno de los mandamientos de las redes sociales. Hay veces que tú mismo puedes ser partícipe de uno pero hay que reconocer que se puede disfrutar de ellos simplemente sentándose frente a la pantalla a observar cómo evolucionan los acontecimientos.

LOL: En inglés, 'Lots of laughs', hace referencia a cuando algo te hace mucha gracia. Pero mucha, mucha, ¿eh? Tanta que unos simples 'jajajaja' no son suficientes. Puede tener múltiples variantes dependiendo de la gracia que te haya hecho algo (LOLAZO, LMAO, LMFAO...) o también puede ir acompañado de muchos emoticonos o 'XD'.

HATER: Persona que odia algo y va a hacer todo lo posible para que te enteres. Quizás incluso tú seas la 
cosa a odiar. Sí, tú. Por algún motivo le caes mal y le va a caer mal todo lo que digas, hagas o publiques porque tiene una fijación maliciosa sobre ti y no vas a poder hacer nada para quitársela. Aun así, para algunos es un goal tener muchos haters. ¿Ves? Ya lo vas pillando.

TROLL: Persona que se dedica a refunfuñar por redes sobre algo que no le gusta sin dar muchos detalles al respecto. Pueden ser tan molestos como una plaga de hormigas en una tranquila tarde de picnic.

STALKEAR: Verbo de primera conjugación, abrir el perfil de la red social de alguien y buscarle hasta la cartilla de nacimiento. Lo que viene siendo el cotilleo de toda la vida. ¿Qué habrá publicado en Instagram hace 1 año? ¿Tendrá más fotos de su amigo en su Twitter? ¿Puso en su Facebook dónde estuvo la semana pasada? Cuidado con stalkear que puede llegar a ser adictivo.

BLESSED: Es un estado de ánimo provocado por algo que te emociona particularmente y por el que te sientes bendecido. No confundir con living, que tiene un punto más de euforia. Blessed es algo más espiritual, más del alma, como cuando después de una noche de fiesta llegas a casa, te quitas los zapatos y te tiras en el sofá.

GOAL: Un mérito que has conseguido en la vida, como conseguir el trabajo de tus sueños, conseguir una prenda en las rebajas que te guste y sea de tu talla o abrir fácilmente un abre fácil.

\section{Referencias}

[1] Caraher, L. (s.f.). Millenials en la oficina. Paidos.

[2] Fall. (2009).

[3] Forbes. (2016). Los millennials se preparan para trabajar durante toda su vida. Forbes.

[4] Hernández Sampieri, R. F. (2014). Metodología de la Investigación (6 ed.). México, México: McGraw Hill.

[5] Haro, J. M. (2017). Millennials La generación emprendedora. España: Ariel.

[6] Herrero, R. (18 de septiembre de 2015). Atraer y retener a los profesionales millennial el management del futuro. Obtenido de https://www.robertwalters.com/content/dam/robert-

walters/corporate/news-and-pr/files/whitepapers/robert-walters-spainattracting-and-retaining-millennials.pdf 